\title{
Anthracnose Fruit and Root Necrosis of Strawberry Are Caused by a Dominant Species Within the Colletotrichum acutatum Species Complex in the United States
}

\author{
Nan-Yi Wang, Bruna B. Forcelini, and Natalia A. Peres ${ }^{\dagger}$ \\ Gulf Coast Research and Education Center, Institute of Food and Agricultural Sciences, University of Florida, Wimauma, FL 33598 \\ Accepted for publication 1 March 2019.
}

\begin{abstract}
Strawberry anthracnose fruit rot and root necrosis, caused by Colletotrichum acutatum, are primary limiting factors in fruit production fields in the United States. Recent research focusing on the phenotypic and genetic characteristics of this species has shed light on the diversity of the $C$. acutatum species complex. In this study, we performed multilocus sequence analysis of four genetic loci to characterize 217 C. acutatum isolates collected over a 23-year period from symptomatic plant tissues of strawberry from six different states. The results revealed two Colletotrichum spp. (C. nymphaeae and C. fioriniae), with $97.7 \%$ of the isolate collection (212 of 217) belonging to $C$. nymphaeae as a dominant clonal linage, regardless of the isolation source. No correlation between species groups and geographical origins of the isolates was observed. Further sequence comparison between historical and contemporary isolates showed the same populations being widely distributed throughout the strawberry

nurseries and production fields in the United States and Canada. Subsequently, a subset of 12 isolates representing different quinoneoutside inhibitor fungicide resistance profiles from root or fruit tissue of strawberry was selected for comparison of pathogenicity on strawberry. In this test, isolates of different resistance groups or different isolation sources exhibited a similar degree of aggressiveness and caused indistinguishable symptoms on strawberry crowns $(P=0.9555$ and 0.7873 , respectively) and fruit $(P=0.1638$ and 0.1141 , respectively), although a significant difference among individual isolates was observed in detached-fruit assays $(P=0.0123)$. Separate pathogenicity tests using isolates of the two species revealed $C$. nymphaeae being more aggressive than $C$. fioriniae in infecting strawberry roots and crowns $(P=0.0073)$. Therefore, given the occurrence and pathogenicity of $C$. nymphaeae, this species is likely the sole cause responsible for strawberry anthracnose in the United States.
\end{abstract}

Florida is the primary producer of fresh winter strawberry (Fragaria $\times$ ananassa) in the United States, accounting for approximately $10 \%$ of the annual strawberry production in the nation (USDA-NASS 2018). The sustainability of the Florida strawberry industry is imperiled by anthracnose fruit rot (AFR) caused by Colletotrichum acutatum J. H. Simmonds (Howard et al. 1992). Under disease-favorable conditions, strawberry growers can experience substantial yield losses greater than $50 \%$ in commercial fields planted with susceptible cultivars (Mertely et al. 2015). Fruit rot and flower blight are the most common symptoms of the disease but other parts of strawberry plants such as leaves, petioles, stolons, roots, and crowns can also be infected by $C$. acutatum (Freeman and Katan 1997; Howard et al. 1992; Peres et al. 2005). Characteristic symptoms of fruit rot appear as dark-brown, sunken necrotic lesions with orange conidial masses produced in acervuli, and the typical symptom of flower blight is brown lesions on petals (Howard et al. 1992; Peres et al. 2005). Root necrosis is characterized by brown to black decayed roots and crown necrosis by reddish to brown necrotic crown tissue after cutting diseased crowns (Freeman and Katan 1997; Howard et al. 1992; Peres et al. 2005). These

†Corresponding author: N. A. Peres; nperes@ufl.edu

Funding: We are grateful for the funding support from the United States Department of Agriculture-National Institute for Food and Agriculture (USDANIFA) Specialty Crop Research Initiative (\#2014-51181-22377) and the USDANIFA Crop Protection and Pest Management (\#2015-70006-24272) programs, as well as the Florida Strawberry Research and Education Foundation.

*The $\boldsymbol{e}$-Xtra logo stands for "electronic extra" and indicates that one supplementary table and two supplementary figures are published online.

The author(s) declare no conflict of interest.

C 2019 The American Phytopathological Society underground infections by $C$. acutatum are often associated with aboveground symptoms such as stunting, wilting, and, in some cases, collapse of plants in the field (Freeman and Katan 1997; Howard et al. 1992; Peres et al. 2005).

Strawberry is a perennial crop but grown annually in Florida due to its warm climate (Brown 2003). The success of this annual production system largely relies on transplants produced remotely from the areas of fruit production fields in high-altitude regions in California and North Carolina and cold-weather locations such as Canada (Brown 2003). Smaller nurseries often obtain planting stock from larger ones for propagation. The interconnectedness among nurseries and between nurseries and fruit fields in Florida creates a path for transmission of microbial pathogens on quiescently infected strawberry transplants (Browne and Bhat 2011; Freeman et al. 2002; Maas et al. 1995; Oliveira et al. 2017; Peres et al. 2005; Ureña-Padilla et al. 2001). Given that the inoculum of $C$. acutatum survives neither on plant residue nor in the soil between seasons in Florida (Ureña-Padilla et al. 2001), the reintroduction of the pathogen on contaminated transplants represents the primary source of inoculum for anthracnose epidemics in the fruit production fields in Florida (Peres et al. 2005; Ureña-Padilla et al. 2001). Standard disease management practices using single- and multiple-site fungicides can be effective in disease control but several outbreaks of anthracnose have been reported in the past two decades (N. A. Peres, unpublished). The most striking case occurred during the 2013-14 strawberry season when, for the first time, C. acutatum isolates resistant to quinone-outside inhibitor (QoI) fungicides were identified and linked back to the transplants from specific strawberry nurseries (Forcelini and Peres 2018; Forcelini et al. 2016).

Some degree of morphological and genetic variation has been found within the species of $C$. acutatum associated with strawberry anthracnose in different geographic regions (Baroncelli et al. 2015; 
Denoyes-Rothan et al. 2003; Freeman and Katan 1997; Garrido et al. 2008; MacKenzie et al. 2009; Ureña-Padilla et al. 2002). Denoyes-Rothan et al. (2003) identified at least two distinct groups of $C$. acutatum populations in strawberry through random amplified polymorphic DNA markers and the ribosomal DNA internal transcribed spacer (ITS) sequences but, shortly thereafter, this number increased to five according to the study of Sreenivasaprasad and Talhinhas (2005). Previous studies in Florida reported that the large majority of the historic populations of $C$. acutatum associated with strawberry from different geographic locations were genetically uniform, with very little variation (MacKenzie et al. 2009; Ureña-Padilla et al. 2002). Our observation of the morphological similarity of the contemporary C. acutatum isolates to the historic populations collected in the early 2000s and 1990s from strawberry fields in Florida seems to be in agreement with that evidence.

Recently, with the aid of multilocus sequence analysis using six different genomic loci, Damm et al. (2012) separated the C. acutatum populations into a species complex containing 31 new Colletotrichum spp., of which at least 6 species were associated with strawberry. Considering the escalating movement of the C. acutatum populations on quiescently infected transplants across the states and nations and the increase in area planted, we would indeed expect high genetic diversity in our C. acutatum populations. Additionally, although the $C$. acutatum isolates collected from either fruit or root tissue of strawberry as well as isolates with different resistance levels to QoI fungicides were morphologically indistinguishable, it is not known whether these isolates are derived from the same population with genetic similarity or whether they are equally aggressive on different strawberry tissue. Therefore, the objectives of the present study were to (i) determine the phylogenetic relatedness of $C$. acutatum species-complex isolates collected during a 23-year period from various geographic regions of North America and from different symptomatic organs, (ii) identify which species within the $C$. acutatum complex were prevalent across the nation using multilocus sequence analysis, (iii) determine the difference in pathogenicity between fruit and root isolates of strawberry with different resistance levels to QoI fungicides, and (iv) determine the difference in pathogenicity between different Colletotrichum spp. The knowledge gained from the study will improve our understanding of the genetic relatedness of Colletotrichum populations within the C. acutatum complex associated with anthracnose of strawberry in the United States.

\section{MATERIALS AND METHODS}

Fungal isolates, growth conditions, and culture characteristics. In total, 217 C. acutatum species-complex isolates collected from strawberry nurseries or fruit production fields across the nation between 1994 and 2016 were used in this study. These included 183 isolates collected in Florida, 12 from California, 9 from Louisiana, 7 from Virginia, 3 from North Carolina, and 3 from Georgia (Table 1; Supplementary Table S1). Except for those kindly provided by other researchers, isolates were recovered from symptomatic plant organs (fruit, crowns, root, petioles, or flowers) on general isolation (GI) medium (potato dextrose agar [PDA] amended with ampicillin at $250 \mathrm{mg} /$ liter and streptomycin at $100 \mathrm{mg} / \mathrm{liter}$ ). After observing fungal colonies on GI plates, isolates were further identified morphologically according to colony and conidial characteristics (Denoyes and Baudry 1995) and, if confirmed, single spored on $1.5 \%$ water agar. Of these isolates, 136 were collected from strawberry fruit, 34 from crowns, 29 from roots, 17 from petioles, and 1 from a flower. Fungal cultures were maintained regularly on half-strength PDA (1/2 PDA) (Oxoid, ThermoFisher Scientific, Wilmington, DE, U.S.A.) dishes at room temperature under continuous light. For long-term storage at $-20^{\circ} \mathrm{C}$, cultures were grown on sterile filter paper (P5; ThermoFisher Scientific) placed on $1 / 2$ PDA at the abovementioned conditions until the filter paper was fully covered with fungal mycelia (Fong et al. 2000). The filter paper was air dried in a laminar flow hood overnight before being stored in sterile coin envelopes in airtight plastic containers with Drierite (W.A. Hammond Drierite Company, Xenia, OH, U.S.A.) at $-20^{\circ} \mathrm{C}$ until use (Fong et al. 2000). In addition, sporulating fungal material was stored in $30 \%$ sterile glycerol at $-80^{\circ} \mathrm{C}$ as backup cultures.

Genomic DNA extraction, gene amplification, and sequencing. Genomic DNA of $C$. acutatum species-complex isolates was extracted according to procedures described in the study of Forcelini et al. (2016). Briefly, fungal mycelia collected from 7 -day-old cultures grown on 1/2 PDA at room temperature under continuous light were transferred to $2-\mathrm{ml}$ screw-cap microfuge tubes with $500 \mu \mathrm{m}$ Garnet and a 6-mm Zirconium Grinding Satellite (SPEX Sample Prep, Metuchen, NJ, U.S.A.). After flash-freezing in liquid nitrogen, mycelia were pulverized twice at $1,500 \mathrm{~min}^{-1}$ for 2 min using a 1600 MiniG automated tissue homogenizer (SPEX Sample Prep) and subjected to DNA extraction using the FastDNA kit (MP Biomedicals, Santa Ana, CA, U.S.A.) according to the manufacturer's instructions. The resulting DNA samples were quantified using a NanoDrop 8000 spectrophotometer (ThermoFisher Scientific) and, if the absorbance ratio of 260/280 nm ranged between 1.7 and 1.9, diluted to the concentration of $25 \mu \mathrm{g} / \mathrm{ml}$ for subsequent gene amplification and sequencing.

To characterize genetic diversity of isolates in the $C$. acutatum species complex, four genomic loci were selected according to Damm et al. (2012), including the ribosomal DNA ITS region and partial genes of $\beta$-tubulin (TUB2), glyceraldehyde-3-phosphate dehydrogenase (GAPDH), and chitin synthase 1 (CHS1). The ITS region was amplified using ITS-1F/ITS-4 primers (Gardes and Bruns 1993; White et al. 1990), TUB2 using TUB-2Fd/Bt-2b or TUB-2Fd/TUB-4Rd primers (Glass and Donaldson 1995; Woudenberg et al. 2009), GAPDH by GDF1/GDR1 primers (Guerber et al. 2003), and CHS1 by CHS-79F/CHS-354R primers (Carbone and Kohn 1999). Polymerase chain reactions (PCR) were performed in a total volume of $25 \mu \mathrm{l}$ containing $1 \mu \mathrm{l}$ of genomic DNA, $0.2 \mu \mathrm{M}$ each primer, $200 \mu \mathrm{M}$ dNTP mix, $1.5 \mathrm{mM} \mathrm{MgCl} 2,1 \times$ Colorless GoTaq Flexi buffer, and $1 \mathrm{U}$ of GoTaq G2 Hot Start polymerase (Promega Corp., Madison, WI, U.S.A.). PCR cycling conditions in a Bio-Rad T100 thermal cycler (Bio-Rad, Hercules, CA, U.S.A.) were as follows: initial denaturation at $95^{\circ} \mathrm{C}$ for $2 \mathrm{~min}$; 40 cycles of amplification at $94^{\circ} \mathrm{C}$ for $30 \mathrm{~s}, 52^{\circ} \mathrm{C}$ for $30 \mathrm{~s}\left(60^{\circ} \mathrm{C}\right.$ for TUB-2Fd/ TUB-4Rd primers), and $72^{\circ} \mathrm{C}$ for $45 \mathrm{~s}$; with a final extension at $72^{\circ} \mathrm{C}$ for $5 \mathrm{~min}$. Subsequently, PCR products were electrophoresed on $1.5 \%$ agarose gels with $1 \times$ GelRed Nucleic Acid Gel Stain (Biotium, Fremont, CA, U.S.A.) in Tris-acetate-EDTA buffer (40 mM Tris-acetate and $0.1 \mathrm{mM}$ EDTA) at $5 \mathrm{~V} / \mathrm{cm}$ for $1 \mathrm{~h}$, visualized using a Bio-Rad Gel Doc EZ imager (Bio-Rad), then sequenced bidirectionally at GENEWIZ (South Plainfield, NJ, U.S.A.). The quality of sequence data was determined using Chromas (version 2.6; Technelysium, South Brisbane, Queensland, Australia) before aligning sequences.

Phylogenetic analysis. Multiple sequence alignment was performed using the MUSCLE algorithm with default settings implemented in MEGA7 (version 7.0.20) (Kumar et al. 2016). To evaluate whether the four sequenced genes were congruent and could be combined, maximum-likelihood analyses were performed for each gene in MEGA7 with subtree pruning and regrafting as the branch swapping algorithm, 1,000 bootstrap replicates, and complete deletion to remove all alignment gaps. The best-fit substitution model was determined separately according to the Bayesian Information Criterion in the model test module of MEGA7. The resulting tree topologies were compared visually (Mason-Gamer and Kellogg 1996). Subsequently, a maximumlikelihood analysis was performed for the dataset of concatenated sequences using MEGA7 according to the abovementioned parameters with the specific use of the Kimura two-parameter and $\gamma$ distribution (eight categories) as the substitution model (Kimura 1980). For additional comparison, the Markov Chain 
Monte Carlo (MCMC) algorithm implemented in MrBayes (version 3.2.6) (Ronquist et al. 2012) was used to generate a phylogenetic tree showing Bayesian probabilities for the concatenated sequence dataset. The MCMC analysis was run with the same substitution model as above for a total of $1,000,000$ generations with the sample frequency of 100 generations to reach a $P$ value $<0.01$. Two parallel searches were included in the analysis, each composed of four chains (one cold and three heated chains with the temperature parameter set to 0.2 ). The first $25 \%$ of generations were discarded as burn-in, and the resulting tree with posterior probabilities was viewed in Mesquite (version 3.40) (Maddison and Maddison 2018). All sequences derived in this study were deposited in GenBank and the alignments and trees in TreeBASE (http:// purl.org/phylo/treebase/phylows/study/TB2:S23514).

Pathogenicity tests. To determine whether isolates obtained from either fruit or root tissue of strawberry were equally aggressive, six arbitrarily selected isolates per tissue were used, including three resistant and three sensitive to QoI fungicides. Pathogenicity tests were conducted on detached fruit and transplants of cultivar Florida Radiance (Chandler et al. 2009; Forcelini et al. 2016, 2018). The inoculation experiments were conducted in the laboratory and greenhouse of the Gulf Coast
Research and Education Center (GCREC) at the University of Florida (Wimauma, FL).

Detached fruit inoculation. Immature fruit that were in transition from green to white and had receptacles with chlorophyll were harvested from experimental fields at GCREC. After sepals were removed, fruit were surface disinfested for $6 \mathrm{~min}$ in $0.06 \%$ (vol/vol) sodium hypochlorite $(\mathrm{NaOCl} ; 1 \%$ household bleach), then rinsed twice for $1 \mathrm{~min}$ each with deionized water. Three fruit per isolate, plus a water control, were placed in clean egg cartons inside clear plastic containers and air dried in a laminar flow hood for $20 \mathrm{~min}$, including $5 \mathrm{~min}$ of ultraviolet treatment. Deionized water $(100 \mathrm{ml})$ was added to the bottom of each container to maintain the humidity inside the containers. For inoculation, conidia of isolates were collected from 7- to 10-day-old cultures grown on 1/2 PDA (Oxoid) under continuous light at room temperature, followed by filtering through eight layers of cheesecloth (ThermoFisher Scientific) and adjusting the concentrations to $10^{6}$ conidia/ml using a hemacytometer. Then, fruit were inoculated with a $20-\mu l$ droplet of either conidial suspensions or sterile deionized water as controls. Closed plastic containers with inoculated fruit were incubated in a growth room at $25^{\circ} \mathrm{C}$ with continuous light for 7 days. Disease severity was assessed as the percentage of fruit area with AFR symptoms. Each

TABLE 1. Summary of Colletotrichum acutatum species-complex isolates sequenced in this study

\begin{tabular}{|c|c|c|c|c|c|c|c|c|}
\hline \multirow[b]{2}{*}{ Geographic origin ${ }^{\mathrm{b}}$} & \multirow[b]{2}{*}{ Nursery source ${ }^{c}$} & \multirow[b]{2}{*}{ Organ } & \multirow[b]{2}{*}{ Year } & \multirow[b]{2}{*}{ Species } & \multicolumn{4}{|c|}{ Number of isolates ${ }^{\mathrm{a}}$} \\
\hline & & & & & Seq & QoI-R & QoI-MR & QoI-S \\
\hline \multirow[t]{3}{*}{ California } & California & Fruit & 2016 & C. nymphaeae & 5 & 1 & 0 & 4 \\
\hline & $\ldots$ & Petiole & 2016 & C. nymphaeae & 5 & 2 & 0 & 3 \\
\hline & N/A & Fruit & 2016 & C. nymphaeae & 2 & 2 & 0 & 0 \\
\hline \multirow[t]{28}{*}{ Florida } & California & Crown & 2013, 2014 & C. nymphaeae & 2 & 1 & 0 & 1 \\
\hline & $\ldots$ & Fruit & $1995,2011,2016$ & C. nymphaeae & 13 & 1 & 0 & 12 \\
\hline & $\ldots$ & Root & 2001 & C. nymphaeae & 1 & 0 & 0 & 1 \\
\hline & Massachusetts & Fruit & 1997 & C. nymphaeae & 1 & 0 & 0 & 1 \\
\hline & Michigan & Crown & 2001 & C. nymphaeae & 1 & 0 & 0 & 1 \\
\hline & $\ldots$ & Root & 2001 & C. nymphaeae & 1 & 0 & 0 & 1 \\
\hline & North Carolina & Crown & 1997, 2002, 2003, 2013 & C. nymphaeae & 7 & 4 & 0 & 3 \\
\hline & $\ldots$ & Fruit & 1995,2014 & C. nymphaeae & 2 & 1 & 0 & 1 \\
\hline & $\ldots$ & Petiole & 2014 & C. nymphaeae & 1 & 0 & 0 & 1 \\
\hline & $\ldots$ & Root & 2013,2014 & C. nymphaeae & 17 & 13 & 1 & 3 \\
\hline & $\ldots$ & & 2013 & C. fioriniae & 1 & 0 & 0 & 1 \\
\hline & Nova Scotia & Crown & 2002, 2003 & C. nymphaeae & 2 & 0 & 0 & 2 \\
\hline & $\ldots$ & Fruit & $1995,2002,2014$ & C. nymphaeae & 6 & 4 & 0 & 2 \\
\hline & $\ldots$ & & 2013 & C. fioriniae & 2 & 0 & 0 & 2 \\
\hline & $\ldots$ & Petiole & 2002 & C. nymphaeae & 1 & 0 & 0 & 1 \\
\hline & $\ldots$ & Root & 2001, 2002 & C. nymphaeae & 2 & 0 & 0 & 2 \\
\hline & Ontario & Crown & 2002, 2013 & C. nymphaeae & 7 & 6 & 0 & 1 \\
\hline & $\ldots$ & Fruit & 2014 & C. nymphaeae & 1 & 1 & 0 & 0 \\
\hline & $\ldots$ & Root & 2013 & C. nymphaeae & 2 & 2 & 0 & 0 \\
\hline & Québec & Crown & 1996, 2001, 2002, 2003, 2013 & C. nymphaeae & 8 & 0 & 1 & 7 \\
\hline & $\ldots$ & Fruit & 1994, 1995, 1997, 2010, 2014 & C. nymphaeae & 15 & 4 & 2 & 9 \\
\hline & $\ldots$ & Petiole & 2001,2003 & C. nymphaeae & 2 & 0 & 0 & 2 \\
\hline & $\ldots$ & Root & 2001 & C. nymphaeae & 2 & 0 & 0 & 2 \\
\hline & N/A & Crown & 1997, 1998, 1999, 2002, 2003 & C. nymphaeae & 7 & 0 & 0 & 7 \\
\hline & $\ldots$ & Fruit & $\begin{array}{c}1996,1999,2001,2007,2008 \\
2010,2011,2013\end{array}$ & C. nymphaeae & 67 & 7 & 0 & 60 \\
\hline & $\ldots$ & & 2013 & C. fioriniae & 2 & 0 & 0 & 2 \\
\hline & $\ldots$ & Petiole & 1998, 1999, 2002 & C. nymphaeae & 7 & 0 & 0 & 7 \\
\hline & $\ldots$ & Root & $\begin{array}{c}2001,2003 \\
2013\end{array}$ & C. nymphaeae & 3 & 0 & 0 & 3 \\
\hline \multirow[t]{2}{*}{ Georgia } & Alabama & Flower & 2016 & C. nymphaeae & 1 & 0 & 0 & 1 \\
\hline & $\ldots$ & Fruit & 2016 & C. nymphaeae & 2 & 1 & 0 & 1 \\
\hline \multirow[t]{2}{*}{ Louisiana } & California & Fruit & 2016 & C. nymphaeae & 7 & 5 & 0 & 2 \\
\hline & Québec & Fruit & 2016 & C. nymphaeae & 2 & 1 & 0 & 1 \\
\hline \multirow[t]{2}{*}{ North Carolina } & California & Fruit & 2016 & C. nymphaeae & 2 & 1 & 0 & 1 \\
\hline & N/A & Petiole & 2016 & C. nymphaeae & 1 & 0 & 0 & 1 \\
\hline Virginia & N/A & Fruit & 2016 & C. nymphaeae & 7 & 3 & 0 & 4 \\
\hline
\end{tabular}

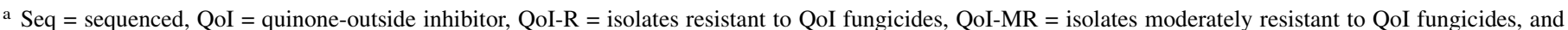
QoI-S = isolates sensitive to QoI fungicides. The QoI-resistance profiles of $C$. acutatum species-complex isolates were determined according to the protocol described by Forcelini et al. (2016).

b Geographical region where isolate was collected.

c Geographical region where plant was produced. N/A = not available. 
container was considered a replicate, and four replicates per isolate were used. The entire experiment was performed twice.

Root inoculation. Four green-top bare-root transplants of Florida Radiance per isolate were root inoculated by dipping for $15 \mathrm{~s}$ in conidial suspensions at $10^{6}$ conidia/ml, prepared as above. Another set of four plants dipped in sterile deionized water served as controls. After inoculation, plants were potted in 1-liter plastic pots filled with potting soil containing 55 to $65 \%$ Canadian sphagnum peat moss (Metro-Mix 820; Sun Gro Horticulture, Agawam, MA, U.S.A.) in a greenhouse, placed randomly on a table, and misted with tap water through misting nozzles for $6 \mathrm{~s}$ every $5 \mathrm{~min}$. Weekly fertilization (Miracle-Gro 24:8:16; Scotts, Marysville, OH, U.S.A.) was applied throughout the experiment, and runners and fruit were removed regularly. Disease progression was evaluated weekly from the fourth to sixth week after inoculation using the scale of Denoyes and Baudry (1995), where $0=$ no lesions, $0.5=$ lesion just visible, $1.0=$ single developed lesion, $1.5=$ two lesions, $2.0=$ at least two leaves or petioles with expanded lesions, $2.5=$ stunted plant but not wilted, $3.0=$ beginning to wilt, $3.5=$ two wilted leaves, $4.0=$ most leaves wilted, $4.5=$ all leaves wilted but slightly green, and $5.0=$ dead plant. Only the final rating of disease severity at the sixth week is reported here. Each of the four inoculated plants represented a replicate and the experiment was repeated three times from March to December 2016.

To determine the aggressiveness of different Colletotrichum spp. identified in this study (i.e., C. nymphaeae and C. fioriniae), different sets of five isolates per species were tested on detached fruit and transplants. The experimental procedures were the same as mentioned above, except for the use of eight fruit per isolate for fruit inoculations and the use of cold-stored frigo transplants for root inoculation due to the unavailability of green-top bare-root transplants at the time when inoculation was performed during February to April 2018. The frigo transplants were first planted in 1-liter plastic pots filled with potting soil in the greenhouse for approximately 4 weeks, after which plants with at least three fully expanded leaves were root inoculated and evaluated as above. Experiments of fruit and root inoculation were performed twice and three times, respectively.

94-11, 95-58E2, 95-59F, 95-60B, 95-61C, 96-65, 96-90, 96-93, 96-95, 97-51A, 97-93, 97-122, 98-1, 98-15, 98-55, 98-296, 99-16, 99-70, 99-100, 99-149, 01-118, 01-119, 01-120, 01-121, 01-122, 01-123, 01-130, 01-131, 01-133, 01-178, 01-213, 02-153, 02-154, 02-157, 02-158, 02-159, 02-160, 02-162, 02-163, 02-164, 02-165, 02-179, 02-180, 03-2, 03-3, 03-4, 03-5, 03-14, 03-15, 03-16, 03-31, 03-32, 07-8, 07-9, 07-10, 07-11,07-12, 07-14, 07-15, $07-16,07-17,07-18,08-11,08-12,08-13,08-14,08-15,08-16,08-17,08-18,08-19,08-20$, $08-23,08-24,08-25,08-26,08-27,08-28,08-29,08-30,08-31,08-32,10-5,10-6,10-7$, $10-8,10-9,10-10,10-11,10-12,10-13,10-14,10-15,10-16,10-17,10-18,10-19,10-20$, $10-21,11-91,11-92,11-93,11-94,11-95,11-96,11-97,11-98,11-99,11-100,11-101,11-102$, $11-103,11-104,11-112,11-113,11-114,11-115,11-116,11-117,11-118,11-119,13-139$, $13-140,13-141,13-142,13-143,13-144,13-148,13-458,13-459,13-462,13-463,13-464$, $13-465,13-466,13-467,13-468,13-469-1,13-469-2,13-470,13-471,13-472,13-473$, $13-374,13-475,13-476,13-477,13-478,13-479,13-480,13-483,13-484,13-487,13-488$, 13-489, 13-492, 13-494, 14-57, 14-58, 14-61, 14-79, 14-87, 14-141, 14-142, 14-143, 14-144, $14-145,14-686,14-687,14-688,14-692,14-697,14-699,16-7,16-28,16-32,16-38,16-49$, $16-65,16-114,16-115,16-130,16-131,16-144,16-147,16-157,16-161,16-170,16-180$,

$16-187,16-196,16-200,16-204,16-207,16-211,16-221,16-230,16-240,16-242,16-245$, $90 / 1.0016-254,16-258,16-259,16-297,16-301,16-307,16-314,16-318,16-320$

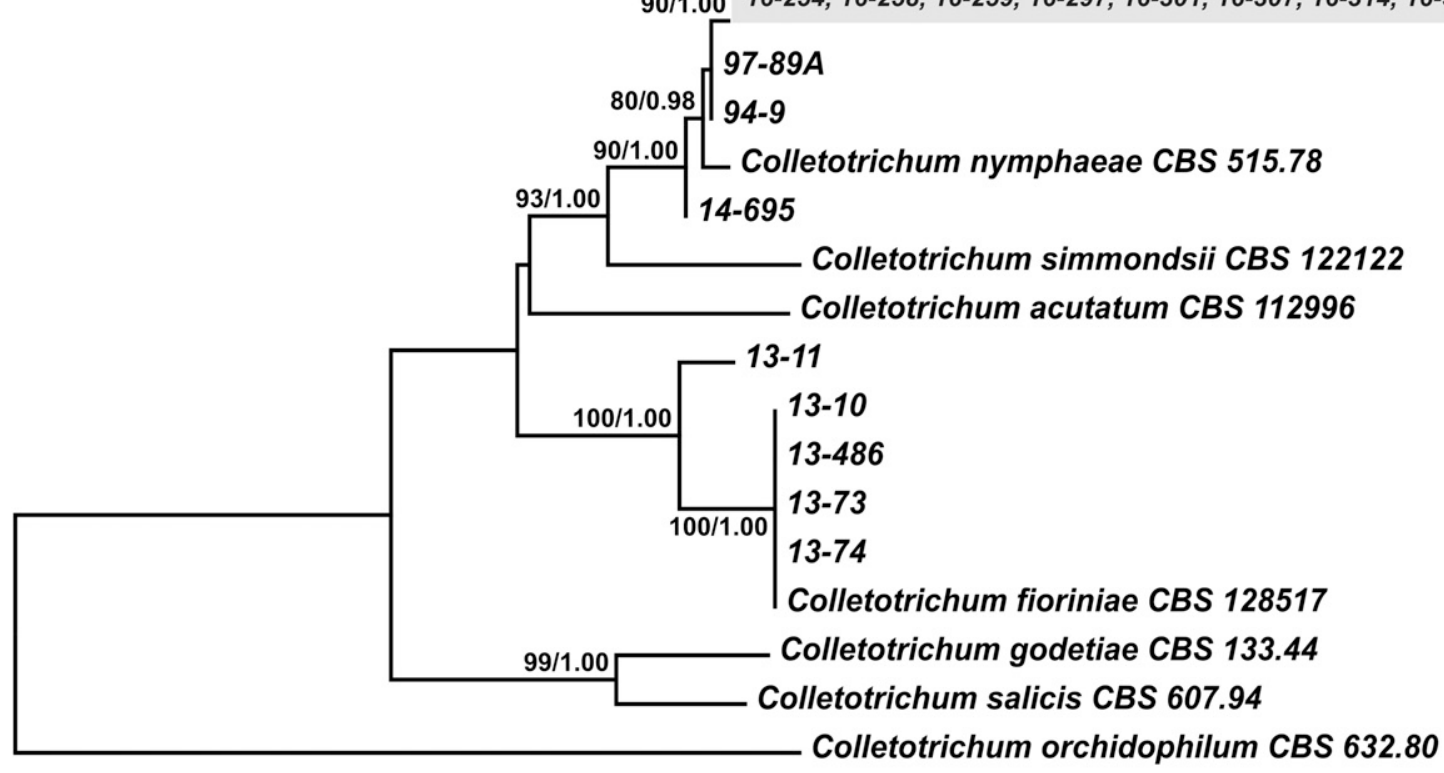

\section{$\check{0.01}$}

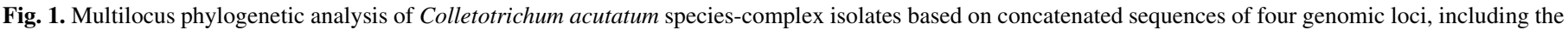

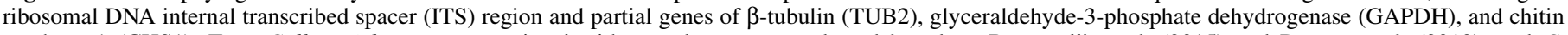

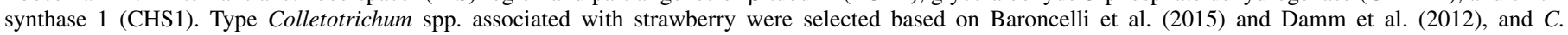

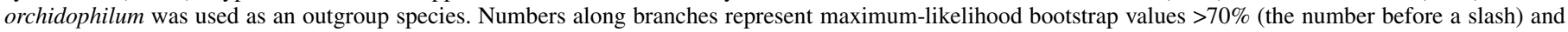

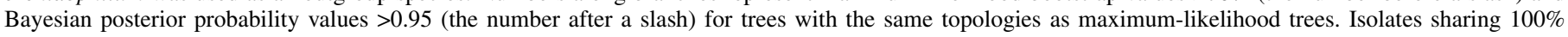

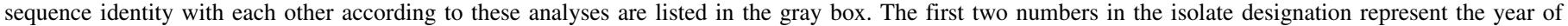
isolation (i.e., 94-11 was collected in 1994). 
Data analysis. Data of disease severity on fruit were analyzed with a generalized linear mixed model using PROC GLIMMIX of SAS (version 9.4; SAS Institute, Cary, NC, U.S.A.). Tissue, species, or resistance group was considered a fixed effect with a normal distribution, whereas experimental repeat, isolate within the interaction between repeat and the fixed effect, and replicate within the interaction among repeat, isolate, and the fixed effect were considered random effects. When isolate was used as a fixed effect, experimental repeat and replicate within the interaction between repeat and isolate were considered random effects. Differences of least-squares means were used to determine significant differences between resistance groups, tissues, species, or isolates at the $P \leq$ 0.05 level. In addition, the Shapiro-Wilk test was used to examine the normality of the residual distribution of the fruit severity data determined after fitting the model.

A nonparametric approach described by Shah and Madden (2004) to analyze ordinal data in factorial experiments was used for the analysis of the disease severity data on plants as determined with the disease severity scale. The overall effect of $C$. acutatum speciescomplex isolates on disease severity was analyzed using PROC MIXED to compute analysis of variance-type statistics of the ranked data and generate relative effects along with $95 \%$ confidence intervals using the LD_CI macro. The relative effect values and standard errors were calculated based on the respective equations: relative effect $=($ mean rank -0.5$) / N$ and standard error $=($ variance $/$ $N)^{1 / 2}$, where $N$ is the total number of experimental units. The difference between resistance groups, tissues, or species was determined using the CONTRAST statement to specify linear contrasts of the mean ranks. To determine whether the ability of isolates to infect fruit and roots was related, Spearman correlation coefficients between fruit severity and mean ranks derived from the plant severity data were calculated using PROC CORR.

\section{RESULTS}

Phylogenetic analysis and species identification. ITS, TUB2, GAPDH, and CHS1 loci of all tested isolates in the C. acutatum species complex responsible for strawberry anthracnose were successfully amplified and sequenced (Table 1). The PCR amplicons of the ITS, TUB2, GAPDH, and CHS1 regions were approximately $620,520,250$, and $280 \mathrm{bp}$, respectively.

Phylogenetic analyses using the combined data set of these four regions showed that 212 of 217 isolates $(97.7 \%)$ grouped together and were identified as belonging to $C$. nymphaeae, according to clustering with the reference C. nymphaeae type strain CBS 515.78, with a bootstrap value of $90 \%$ and a posterior probability of 1.00 (Fig. 1; Supplementary Fig. S1). The remaining five isolates (2.3\%)

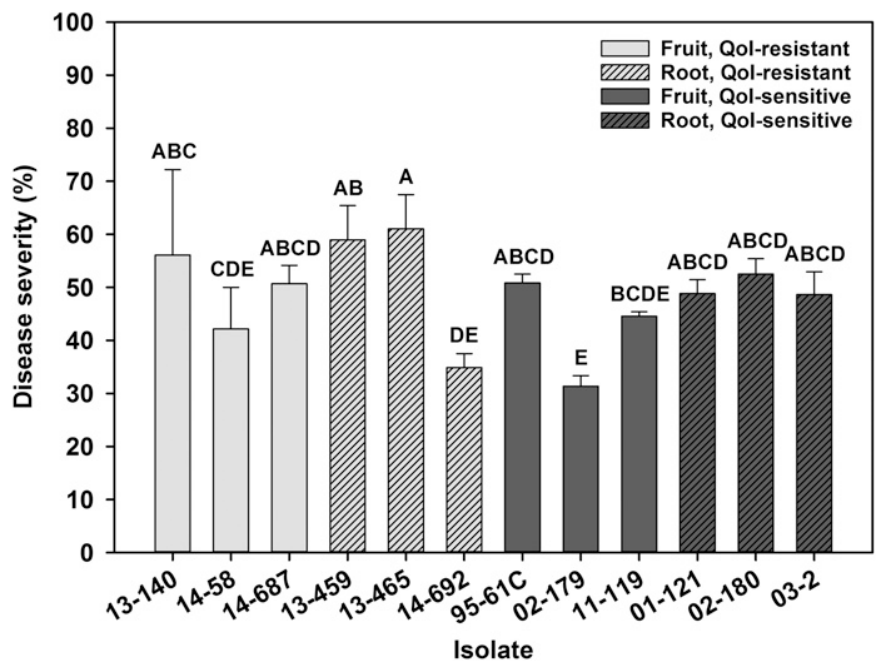

Fig. 3. Disease severity of detached strawberry fruit inoculated with Colletotrichum nymphaeae isolates of different fungicide resistance profiles obtained from either fruit or root tissue of strawberry. Each value represents the mean of two independent experiments with four replicates of each experiment. Error bars present the standard error of the mean. Vertical bars labeled with the same letter are not significantly different from each other according to differences of least-square means $(P \leq 0.05)$.

TABLE 2. Analysis of variance-type statistics based on the mean ranks of disease severity of strawberry plants root inoculated with Colletotrichum nymphaeae isolates of different fungicide resistance profiles obtained from either strawberry fruit or root

\begin{tabular}{lcccc}
\hline & \multicolumn{4}{c}{ Statistics $^{\mathrm{a}}$} \\
\cline { 2 - 5 } Effect & $\mathrm{df}_{\mathrm{Num}}$ & $\mathrm{df}_{\text {Dem }}$ & $F$ & $P$ value \\
\hline Isolate & 9.8 & 31.7 & 0.14 & 0.9985 \\
Fruit versus root & 1 & 31.7 & 0.07 & 0.7873 \\
Resistant versus sensitive & 1 & 31.7 & 0.00 & 0.9555 \\
\hline
\end{tabular}

${ }^{\text {a }}$ Results represent data from four independent experiments; $\mathrm{df}_{\mathrm{Num}}=$ numerator degrees of freedom and $\mathrm{df}_{\mathrm{Dem}}=$ denominator degrees of freedom.
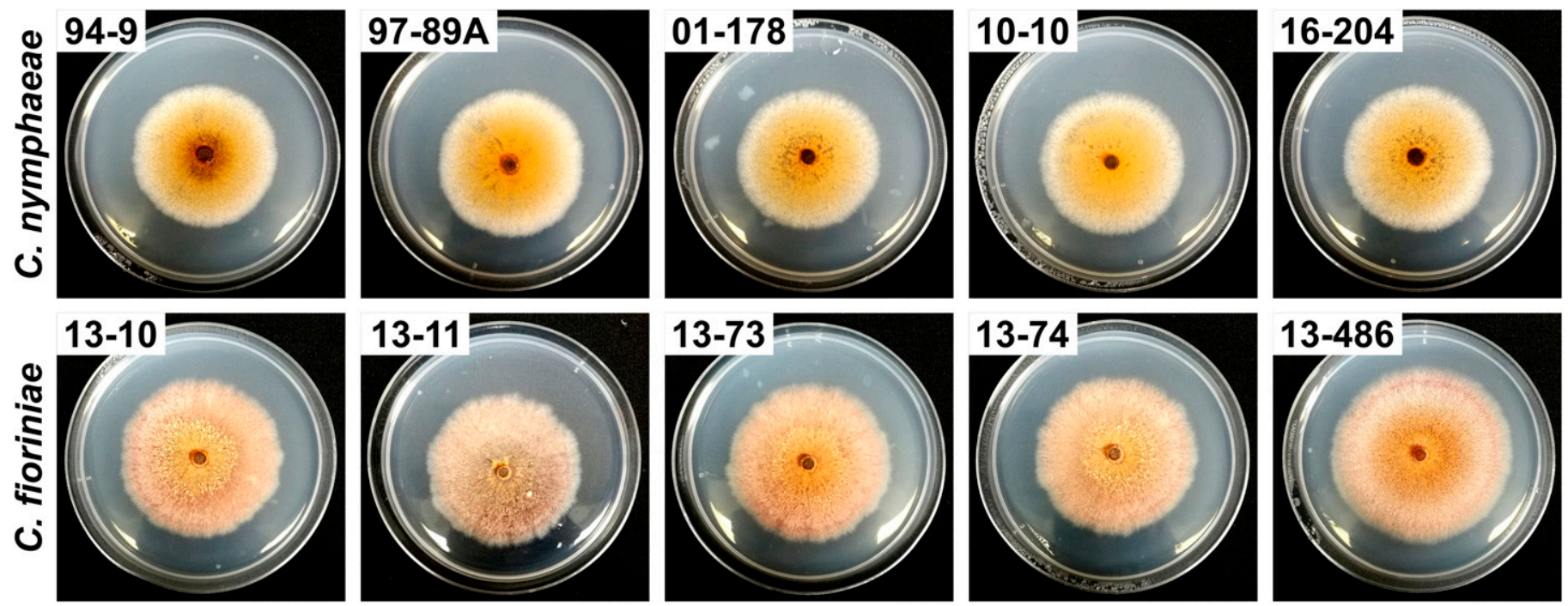

Fig. 2. Colony morphology of representative isolates of various Colletotrichum spp. grown on half-strength potato dextrose agar (1/2 PDA) dishes (100 mm in diameter) for 7 days at room temperature under continuous light. Colletotrichum nymphaeae isolates (upper panel) exhibited one typical color in culture on 1/2 PDA, whereas C. fioriniae isolates (lower panel), also referred to as chromogenic isolates in the literature (Denoyes-Rothan et al. 2003; MacKenzie et al. 2009), produced a different pigment in culture. 
grouped together with the reference type strain of $C$. fioriniae CBS 128517 , with a bootstrap value of $100 \%$ and a posterior probability of 1.00 (Fig. 1). The analyses also revealed three haplotypes of C. nymphaeae and two haplotypes of $C$. fioriniae based on sequence variability. No correlation between species groups and geographical origins of the isolates was observed, and all QoI-resistant isolates were found in the $C$. nymphaeae clade. Among the four regions, the GAPDH locus had the highest variability, with $15.0 \%$ polymorphic sites across the data set (outgroup species excluded). The CHS1 locus ranked second in sequence variability with $4.9 \%$ polymorphic sites, followed by TUB2 and ITS loci with 3.9 and $3.0 \%$ polymorphism in the data set, respectively.

C. nymphaeae isolates formed white-to-gray mycelia and abundant orange conidial masses without pigment production in culture on 1/2 PDA medium (Fig. 2). However, $C$. fioriniae isolates produced red-to-pink chromogenic color in culture (Fig. 2), which is the most characteristic difference between these two Colletotrichum spp. No perithecia were observed in cultures of the two species.

Pathogenicity tests of root and fruit isolates of $C$. nymphaeae with different QoI resistance profiles. Because C. nymphaeae was the dominant Colletotrichum sp. identified in our populations, 12 C. nymphaeae isolates, including 3 isolates per resistance grouporgan combination, were arbitrarily selected for pathogenicity tests on detached fruit and strawberry transplants. All isolates, regardless of their fungicide resistance profiles or isolation sources, caused typical AFR symptoms on detached fruit, with disease severity ranging from 31.4 to $61.0 \%$ (Fig. 3). Although this variability among individual isolates was significant $(P=0.0123)$, the difference between resistance groups or between fruit and root isolates was not significant $(P=0.1638$ and 0.1141 , respectively). During the experimental period, control fruit treated with sterile deionized water remained asymptomatic (data not shown).

In the plant root inoculations, all isolates were pathogenic on strawberry transplants, causing either wilting or plant death after 6 weeks in the greenhouse, whereas water-treated plants remained healthy. The mean ranks of disease severity across isolates ranged from 20.3 to 28.8 and were not significantly different from each other $(P=0.9985)$ (Tables 2 and 3$)$. Similarly, no significant difference was observed between resistance groups $(P=0.9555)$ or between fruit and root isolates $(P=0.7873)$ (Table 2$)$. The correlation between fruit severity and root severity ratings among isolates was not significant $(P=0.1473)$.

Pathogenicity tests of different Colletotrichum spp. Both Colletotrichum spp. identified in the populations were capable of causing decay on detached fruit, although the severity rating was generally low (Fig. 4). Two C. nymphaeae isolates (94-9 and 9789A) showed a significantly higher level of aggressiveness, with disease severity ranging from 13.2 to $15.2 \%$, compared with 1.4 to $3.8 \%$ for $C$. fioriniae isolates $(P=0.0088)$ (Fig. 4). Moreover, a more uniform trend was observed in the plant root assays, with C. nymphaeae isolates producing significantly higher mean disease severity ranks (15.7 to 22.3$)$ compared with $C$. fioriniae isolates (7.5 to 16.0) $(P=0.0073)$ (Table 4$)$. Of these $C$. nymphaeae isolates, 94-9, 01-178, 10-10, and 16-204 appeared to be more aggressive

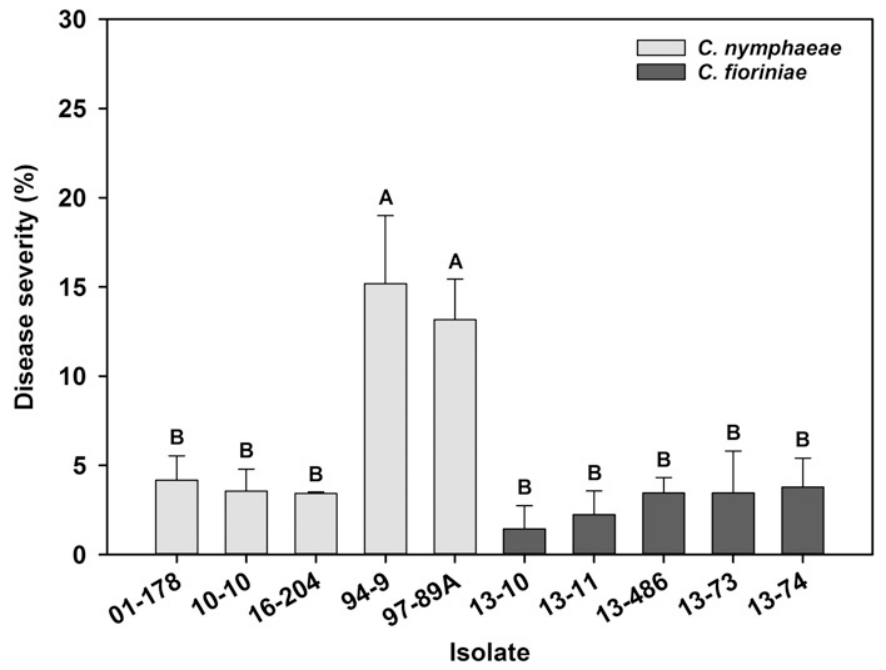

Fig. 4. Disease severity of detached strawberry fruit inoculated with isolates of two Colletotrichum spp. Each value represents the mean of two independent experiments with four replicates of each experiment. Error bars present the standard error of the mean. Vertical bars labeled with the same letter are not significantly different from each other according to differences of least-square means $(P \leq 0.05)$.

TABLE 3. Median, mean ranks, and relative effects along with 95\% confidence intervals (CI) calculated for disease severity of strawberry plants root inoculated with Colletotrichum nymphaeae isolates of different fungicide resistance profiles obtained from either strawberry fruit or root ${ }^{\mathrm{a}}$

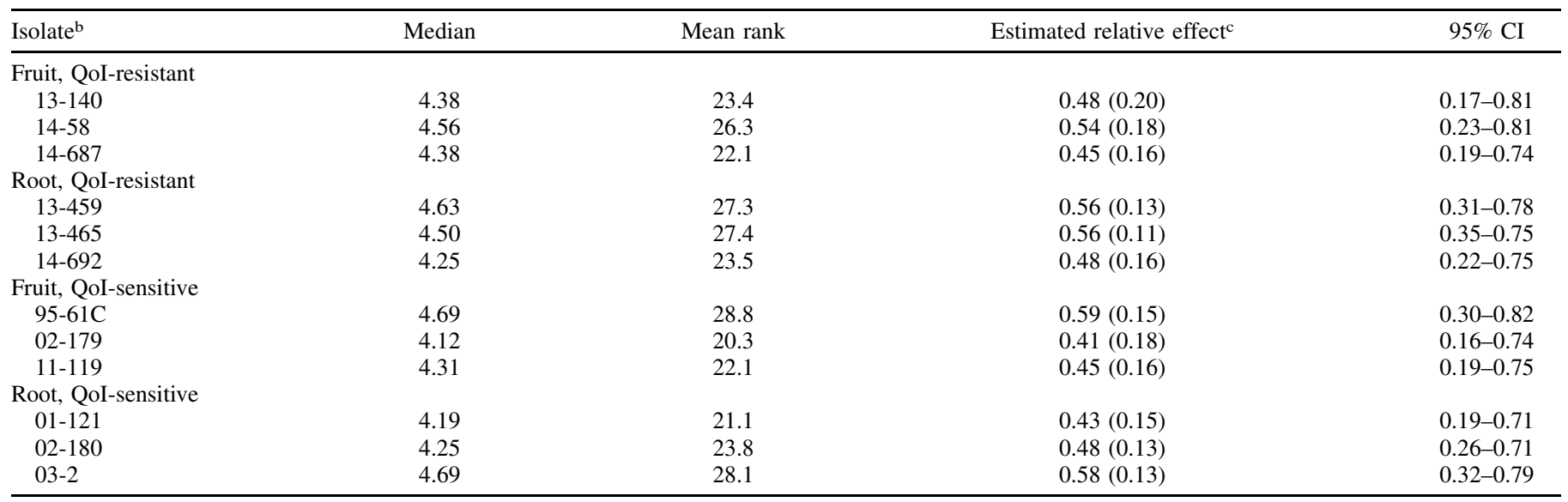

${ }^{a}$ Disease severity was assessed visually according to the scale described by Denoyes and Baudry (1995), where $0=$ no lesions, $0.5=$ lesion just visible, $1.0=$ single developed lesion, $1.5=$ two lesions, $2.0=$ at least two leaves or petioles with expanded lesions, $2.5=$ stunted plant but not wilted, $3.0=$ beginning to wilt, $3.5=$ two wilted leaves, $4.0=$ most leaves wilted, $4.5=$ all leaves wilted but slightly green, and $5.0=$ dead plant. Median values were calculated from the average disease severity rating of four independent experiments. Mean ranks, relative effects, and 95\% CI were determined using the analysis of variance-type statistics with the LD_CI macro (Shah and Madden 2004). Values are means of four independent experiments with four replicates of each experiment.

b Fungicide resistance profiles (QoI = quinone-outside inhibitor) of $C$. nymphaeae isolates obtained from either fruit or root tissue of strawberry samples were determined according to our previous study (Forcelini et al. 2016).

${ }^{c}$ Numbers in parentheses indicate standard errors. 
on strawberry plants (Supplementary Fig. S2). Unlike the previous experiments, some control plants showed wilting of the oldest leaves with ratings between 3.0 and 3.5 at 6 weeks postinoculation, which is likely due to natural senescence of the frigo transplants. The correlation of fruit severity with plant severity rating between the two Colletotrichum spp. was not significant $(P=0.0765)$.

\section{DISCUSSION}

The current evidence suggests that epidemics of strawberry anthracnose in Florida begin with the arrival of $C$. acutatum populations on quiescently infected transplants from nurseries (Freeman et al. 2002; Peres et al. 2005; Ureña-Padilla et al. 2001). The introduction of the pathogen has been found more frequently along with the expansion of planting area in recent years and seems to correlate with anthracnose outbreaks caused by $C$. acutatum in Florida strawberry production fields (Forcelini and Peres 2018; Forcelini et al. 2016) (N. A. Peres, unpublished). Historically, the cause of the disease in the United States was attributed mainly to one C. acutatum population (MacKenzie et al. 2009). In the light of the movement of quiescently infected transplants across states and nations, and the fact that epidemics of crown and root necrosis have recently become more common, we hypothesized an increased level of genetic diversity in the contemporary C. acutatum populations in the United States. However, based on phylogenetic analyses using four genetic loci of 217 C. acutatum species-complex isolates collected over a 23 -year period from multiple states and strawberry organs, our results reject the hypothesis. Instead, our results revealed very little intraspecies variation in the two identified Colletotrichum populations, C. nymphaeae and C. fioriniae, of which C. nymphaeae is the dominant species, accounting for $97.7 \%$ of the tested isolates. Further comparison of our sequence data with those used in previous studies (Denoyes-Rothan et al. 2003; MacKenzie et al. 2009) showed that more recent isolates of each species were genetically identical to the historical ones (data not shown). Our findings, along with the lack of correlation among species, geographical origins of the isolates, and infected organs, indicate that the same population in the $C$. acutatum species complex is widespread across strawberry nurseries and fruit production fields in the United States and Canada, likely through quiescently infected transplants.

Although our analyses only identified $C$. nymphaeae and C. fioriniae to be responsible for strawberry anthracnose in the United States, other species in the $C$. acutatum species complex have been found in other strawberry-growing regions of the world. Baroncelli et al. (2015) reported three different species associated with strawberry production in the United Kingdom, including $C$. nymphaeae, $C$. fioriniae, and $C$. godetiae. A more diverse scenario was found in Belgium, where $C$. nymphaeae represented $33 \%$ of the populations (A2 intraspecific group), whereas C. fioriniae contributed 5\% (A3), C. godetiae 50\% (A4), C. salicis $6 \%$ (A7), and C. acutatum sensu stricto 3\% (A5) (Van Hemelrijck et al. 2010). However, similar to the present study, C. nymphaeae-referred to as the CA-clonal group by DenoyesRothan et al. (2003) or group D by Freeman and Katan (1997)constituted the majority of strawberry anthracnose isolates found in France, Spain, Bulgaria, and Israel (Denoyes-Rothan et al. 2003; Freeman and Katan 1997; Garrido et al. 2008; Jelev et al. 2008). It is unclear why various Colletotrichum spp. associated with strawberry production are present in different geographical regions but not in the United States. Given the international and local trade practices for planting material, and our spatiotemporal collection of isolates from various nursery sources over the years, other species in the C. acutatum species complex, if already present in the United States, should appear in our culture collection.

According to inoculation experiments in 2016, C. nymphaeae isolates from different plant tissues were equally aggressive and caused similar levels of disease severity on detached fruit and strawberry transplants. Similarly, isolates of different QoI resistance profiles did not differ in aggressiveness, as was observed previously by Forcelini et al. (2018). However, some variation in disease severity on detached fruit but not on whole plants was observed among individual isolates. This variation has also been reported in the CA-clonal populations of $C$. acutatum (=C. nymphaeae) by Denoyes-Rothan et al. (2003) and could be attributed to different genetic backgrounds of the isolates rather than isolation sources or fitness penalties, although the tested isolates in the study were genetically identical based on the four loci. In the case of pathogenicity tests in 2018, the two C. nymphaeae isolates 94-9 and 97-89A exhibited a significantly higher level of aggressiveness. Given that these isolates clustered apart from the majority of $C$. nymphaeae isolates tested, the difference in aggressiveness may be characteristic of the two isolates and may not be generalized to the $C$. nymphaeae species. These results may suggest a need for the use of molecular markers with greater genotypic resolution such as simple sequence repeat markers to further differentiate isolates within the species and gain insights into their genetic variation in relation to aggressiveness.

C. nymphaeae and $C$. fioriniae can cause diseases on a wide range of hosts such as apple (Munir et al. 2016), blueberry (MacKenzie et al. 2009), olive (Mosca et al. 2014), peach (Chen et al. 2016), and strawberry (Baroncelli et al. 2015; MacKenzie et al. 2009; Van

TABLE 4. Median, mean ranks, and relative effects along with 95\% confidence intervals (CI) calculated for disease severity of strawberry plants root-inoculated with two Colletotrichum spp. ${ }^{a}$

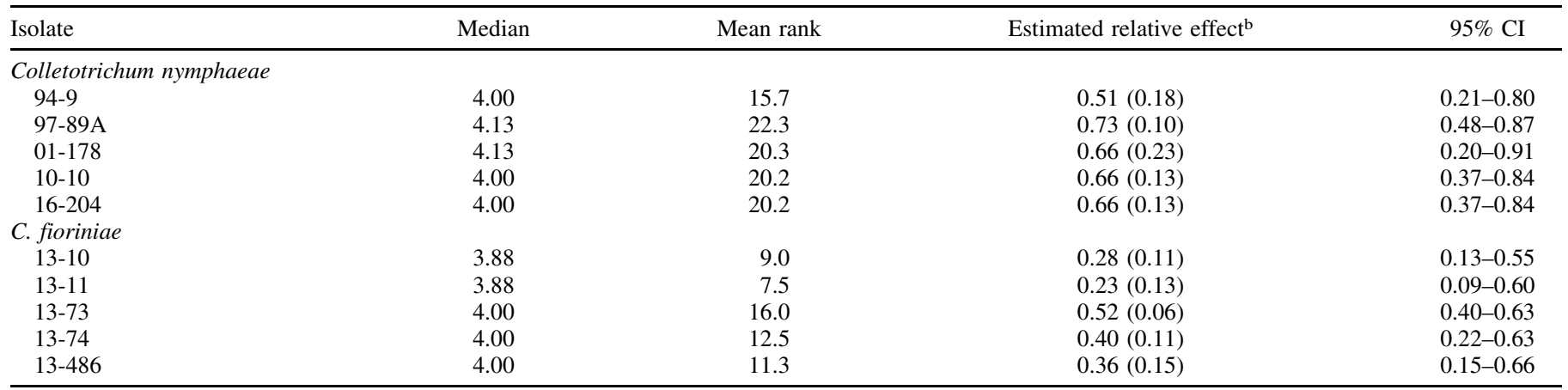

${ }^{a}$ Disease severity was assessed visually according to the scale described by Denoyes and Baudry (1995), where $0=$ no lesions, $0.5=$ lesion just visible, $1.0=$ single developed lesion, $1.5=$ two lesions, $2.0=$ at least two leaves or petioles with expanded lesions, $2.5=$ stunted plant but not wilted, $3.0=$ beginning to wilt, $3.5=$ two wilted leaves, $4.0=$ most leaves wilted, $4.5=$ all leaves wilted but slightly green, and $5.0=$ dead plant. Median values were calculated from the average disease severity rating of four independent experiments. Mean ranks, relative effects, and $95 \%$ CI were determined using the analysis of variance-type statistics with the LD_CI macro (Shah and Madden 2004). Values are means of three independent experiments with four replicates of each experiment.

b Numbers in parentheses indicate standard errors. 
Hemelrijck et al. 2010). In this study, results of the pathogenicity tests with isolates of two different Colletotrichum spp. were consistent with the previous reports, showing that both C. nymphaeae and C. fioriniae are pathogenic to strawberry. Nonetheless, $C$. nymphaeae exhibited higher levels of aggressiveness on strawberry than $C$. fioriniae, as evaluated in the plant root assays. Similar pathogenic superiority of C. nymphaeae (strawberry isolates of $C$. acutatum) over $C$. fioriniae (blueberry isolates of $C$. acutatum) on strawberry has been reported previously by MacKenzie et al. (2009) but this relationship seems host specific because $C$. fioriniae was more aggressive than $C$. nymphaeae on apple (Munir et al. 2016) and blueberry fruit (MacKenzie et al. 2009). Moreover, MacKenzie et al. (2009) found that postbloom fruit drop isolates of $C$. acutatum were more aggressive on citrus flower clusters than other heterologous hosts, and strawberry, Key lime, and leatherleaf isolates only caused diseases on their original hosts after foliar inoculation. Thus, the authors concluded that Colletotrichum spp., while capable of cross-infecting a wide range of hosts, would be more aggressive or, in some cases, the only pathogen on the host or tissue of origin. These findings of specific host-pathogen interactions based on pathogenicity assays might explain, at least in part, the dominance of $C$. nymphaeae isolates responsible for anthracnose of strawberry in the nurseries and production fields in the United States.

In the present study, $C$. fioriniae was found to account for only a small portion of the population from strawberry in Florida. The reason for the occurrence of $C$. fioriniae at such a low frequency on strawberry remains unclear but might be explained by the combination of specific host or tissue preferences and unfavorable climatic conditions in Florida. C. fioriniae has been confirmed to cause strawberry fruit decay but failed to induce foliar symptoms or crown necrosis unless more intense inoculation methods such as crown injection or root dipping are applied (Baroncelli et al. 2015; MacKenzie et al. 2009; Van Hemelrijck et al. 2010). Although quiescent infection on petioles and leaves is part of the life cycle of $C$. fioriniae, this fruit preference might make the pathogen population unsustainable in the absence of fruit, particularly in strawberry nurseries where flower buds and fruit are usually removed during plant production. In Florida, the hot summer climate impedes the survival of C. acutatum populations (Ureña-Padilla et al. 2001). However, this is not the case in other crops in temperate regions. According to Chen et al. (2016), C. fioriniae appeared in a high percentage of symptomatic peach fruit samples in Georgia and South Carolina orchards. Similarly, Munir et al. (2016) found that $71 \%$ (335 of 475) of the pathogen populations from apple orchards of Kentucky belonged to $C$. fioriniae. In the case of blueberry, Smith et al. (1996) found that ripe rot was mostly caused by C. gloeosporioides in Florida whereas, further north in Mississippi with a more temperate climate, the disease was predominantly caused by C. acutatum (sensu lato), including isolates with pink chromogenic color in culture $(=C$. fioriniae $)$. Together, these findings imply that strawberry is not the primary host for $C$. fioriniae, at least in locations with hot summer weather such as Florida; and, therefore, epidemics of strawberry anthracnose are unlikely to be incited by $C$. fioriniae in Florida.

In conclusion, with the aid of multilocus sequence analysis using four genetic loci, we identified two distinct species within the C. acutatum species complex responsible for strawberry anthracnose in the United States: $C$. nymphaeae and $C$. fioriniae. Sequence comparison between historical and contemporary populations showed very little genetic diversity, not as hypothesized, which indicates that the same population is widely spread throughout strawberry nurseries and fruit production fields in the United States and Canada, likely via quiescently infected transplants. Of these two species, $C$. nymphaeae was found to be dominant in the populations and likely is the sole cause of the disease in the United States according to isolation frequency and pathogenicity tests. Considering the recent finding of resistance in $C$. nymphaeae populations to the QoI fungicides (Forcelini et al. 2016) and the fact that resistant isolates have been shown to be equally aggressive and compete well with sensitive isolates (Forcelini et al. 2018), management strategies that reduce the spread of this clonal-QoIresistant population are needed.

\section{ACKNOWLEDGMENTS}

We thank L. T. Carter, M. V. Marin, and R. G. Ruschel for their technical assistance with DNA extraction and inoculation experiments; and P. M. Brannen, R. D. Flanagan, M. J. Hu, K. L. Ivors, B. Poling, and G. Schnabel for kindly providing C. acutatum isolates or symptomatic samples for culture isolation for this study.

\section{LITERATURE CITED}

Baroncelli, R., Zapparata, A., Sarrocco, S., Sukno, S. A., Lane, C. R., Thon, M. R., Vannacci, G., Holub, E., and Sreenivasaprasad, S. 2015. Molecular diversity of anthracnose pathogen populations associated with UK strawberry production suggests multiple introductions of three different Colletotrichum species. PLoS One 10:e0129140.

Brown, M. 2003. Florida strawberry production and marketing. Pages 31-42 in: The Strawberry: A Book for Growers, Others. N. F. Childers, ed. Dr. Norman N. Childers Publications, Gainesville, FL, U.S.A.

Browne, G. T., and Bhat, R. G. 2011. Phytophthora crown and root rot. Calif. Strawb. Comm. 9:1-4.

Carbone, I., and Kohn, L. M. 1999. A method for designing primer sets for speciation studies in filamentous ascomycetes. Mycologia 91:553-556.

Chandler, C. K., Santos, B. M., Peres, N. A., Jouquand, C., Plotto, A., and Sims, C. A. 2009. 'Florida Radiance' strawberry. HortScience 44:1769-1770.

Chen, S. N., Luo, C. X., Hu, M. J., and Schnabel, G. 2016. Sensitivity of Colletotrichum species, including $C$. fioriniae and $C$. nymphaeae, from peach to demethylation inhibitor fungicides. Plant Dis. 100:2434-2441.

Damm, U., Cannon, P. F., Woudenberg, J. H. C., and Crous, P. W. 2012. The Colletotrichum acutatum species complex. Stud. Mycol. 73:37-113.

Denoyes, B., and Baudry, A. 1995. Species identification and pathogenicity study of French Colletotrichum strains isolated from strawberry using morphological and cultural characteristics. Phytopathology 85:53-57.

Denoyes-Rothan, B., Guérin, G., Délye, C., Smith, B., Minz, D., Maymon, M., and Freeman, S. 2003. Genetic diversity and pathogenic variability among isolates of Colletotrichum species from strawberry. Phytopathology 93: 219-228.

Fong, Y. K., Anuar, S., Lim, H. P., Tham, F. Y., and Sanderson, F. R. 2000. A modified filter paper technique for long-term preservation of some fungal cultures. Mycologist 14:127-130.

Forcelini, B. B., and Peres, N. A. 2018. Widespread resistance to QoI fungicides of Colletotrichum acutatum from strawberry nurseries and production fields. Plant Health Prog. 19:338-341. doi:10.1094/PHP-08-18-0050-RS

Forcelini, B. B., Rebello, C. S., Wang, N.-Y., and Peres, N. A. 2018. Fitness, competitive ability, and mutation stability of isolates of Colletotrichum acutatum from strawberry resistant to QoI fungicides. Phytopathology 108: 462-468.

Forcelini, B. B., Seijo, T. E., Amiri, A., and Peres, N. A. 2016. Resistance in strawberry isolates of Colletotrichum acutatum from Florida to quinoneoutside inhibitor fungicides. Plant Dis. 100:2050-2056.

Freeman, S., and Katan, T. 1997. Identification of Colletotrichum species responsible for anthracnose and root necrosis of strawberry in Israel. Phytopathology 87:516-521.

Freeman, S., Shalev, Z., and Katan, J. 2002. Survival in soil of Colletotrichum acutatum and C. gloeosporioides pathogenic on strawberry. Plant Dis. 86: 965-970.

Gardes, M., and Bruns, T. D. 1993. ITS primers with enhanced specificity for basidiomycetes - application to the identification of mycorrhizae and rusts. Mol. Ecol. 2:113-118.

Garrido, C., Carbú, M., Fernández-Acero, F. J., Budge, G., Vallejo, I., Colyer, A., and Cantoral, J. M. 2008. Isolation and pathogenicity of Colletotrichum spp. causing anthracnose of strawberry in south west Spain. Eur. J. Plant Pathol. 120:409-415.

Guerber, J. C., Liu, B., Correll, J. C., and Johnston, P. R. 2003. Characterization of diversity in Colletotrichum acutatum sensu lato by sequence analysis of two gene introns, mtDNA and intron RFLPs, and mating compatibility. Mycologia 95:872-895.

Howard, C. M., Maas, J. L., Chandler, C. L., and Albregts, E. A. 1992. Anthracnose of strawberry caused by the Colletotrichum complex in Florida. Plant Dis. 76:976-981.

Jelev, Z. J., Bobev, S. G., Minz, D., Maymon, M., and Freeman, S. 2008. Characterization of Colletotrichum species causing strawberry anthracnose in Bulgaria. J. Phytopathol. 156:668-677.

Kimura, M. 1980. A simple method for estimating evolutionary rates of base substitutions through comparative studies of nucleotide sequences. J. Mol. Evol. 16:111-120.

Kumar, S., Stecher, G., and Tamura, K. 2016. MEGA7: Molecular evolutionary genetics analysis version 7.0 for bigger datasets. Mol. Biol. Evol. $30: 2725-2729$. 
Glass, N L., and Donaldson, G. C. 1995. Development of primer sets designed for use with the PCR to amplify conserved genes from filamentous ascomycetes. Appl. Environ. Microbiol. 61:1323-1330.

Maas, J. L., Pooler, M. R., and Galleta, G. J. 1995. Bacterial angular leaf spot disease of strawberry: Present status and prospects for control. Adv. Strawb. Res. 14:18-24

MacKenzie, S. J., Peres, N. A., Barquero, M. P., Arauz, L. F., and Timmer, L. W. 2009. Host range and genetic relatedness of Colletotrichum acutatum isolates from fruit crops and leatherleaf fern in Florida. Phytopathology 99:620-631.

Maddison, W. P., and Maddison, D. R. 2018. Mesquite: A modular system for evolutionary analysis. Version 3.40. http://www.mesquiteproject.org/

Mason-Gamer, R., and Kellogg, E. 1996. Testing for phylogenetic conflict among molecular datasets in the tribe Tiriceae (Graminae). Syst. Biol. 45:524-545.

Mertely, J. C., Seijo, T. E., and Peres, N. A. 2015. Evaluation of products for anthracnose and Botrytis fruit rot control in annual strawberry, 2014-2015. Plant Dis. Manage. Rep. 9: Report SMF019. https://www.plantmanagementnetwork. org/pub/trial/PDMR/volume9/abstracts/SMF19.asp

Mosca, S., Nicosia, M. G. L. D., Cacciola, S. O., and Schena, L. 2014. Molecular analysis of Colletotrichum species in the carposphere and phyllosphere of olive. PLoS One 9:e114031.

Munir, M., Amsden, B., Dixon, E., Vaillancourt, L., and Gauthier, N. W. 2016. Characterization of Colletotrichum species causing bitter rot of apple in Kentucky orchards. Plant Dis. 100:2194-2203.

Oliveira, M. S., Amiri, A., Zuniga, A. I., and Peres, N. A. 2017. Sources of primary inoculum of Botrytis cinerea and their impact on fungicide resistance development in commercial strawberry fields. Plant Dis. 101:1761-1768.

Peres, N. A., Timmer, L. W., Adaskaveg, J. E., and Correll, J. C. 2005. Lifestyles of Colletotrichum acutatum. Plant Dis. 89:784-796.

Ronquist, F., Teslenko, M., van Der Mark, P., Ayres, D. L., Darling, A., Höhna, S., Larget, B., Liu, L., Suchard, M. A., and Huelsenbeck, J. P. 2012.
MrBayes 3.2: Efficient Bayesian phylogenetic inference and model choice across a large model space. Syst. Biol. 61:539-542.

Shah, D. A., and Madden, L. V. 2004. Nonparametric analysis of ordinal data in designed factorial experiments. Phytopathology 94:33-43.

Smith, B. J., Magee, J. B., and Gupton, C. L. 1996. Susceptibility of rabbiteye blueberry cultivars to postharvest diseases. Plant Dis. 80:215-218.

Sreenivasaprasad, S., and Talhinhas, P. 2005. Genotypic and phenotypic diversity in Colletotrichum acutatum, a cosmopolitan pathogen causing anthracnose on a wide range of hosts. Mol. Plant Pathol. 6:361-378.

USDA-NASS. 2018. Vegetables: 2017 Summary. United States Department of Agriculture-National Agricultural Statistics Service. https://downloads. usda.library.cornell.edu/usda-esmis/files/02870v86p/5425kd81z/9019s517t/ VegeSumm-02-13-2018.pdf

Ureña-Padilla, A. R., MacKenzie, S. J., Bowen, B. W., and Legard, D. E. 2002. Etiology and population genetics of Colletotrichum spp. causing crown and fruit rot of strawberry. Phytopathology 92:1245-1252.

Ureña-Padilla, A. R., Mitchell, D. J., and Legard, D. E. 2001. Oversummer survival of inoculum for Colletotrichum crown rot in buried strawberry crown tissue. Plant Dis. 85:750-754.

Van Hemelrijck, W., Debode, J., Heungens, K., Maes, M., and Creemers, P. 2010. Phenotypic and genetic characterization of Colletotrichum isolates from Belgian strawberry fields. Plant Pathol. 59:853-861.

White, T. J., Bruns, T. D., Lee, S. B., and Taylor, J. W. 1990. Amplification and direct sequencing of fungal ribosomal RNA genes for phylogenetics. Pages 315-322 in: PCR Protocols: A Guide to Methods and Applications. M. A. Innis, D. H. Gelfand, J. J. Sninsky, and T. J. White, eds. Academic Press, San Diego, CA, U.S.A.

Woudenberg, J. H. C., Aveskamp, M. M., De Gruyter, J., Spiers, A. G., and Crous, P. W. 2009. Multiple Didymella teleomorphs are linked to the Phoma clematidina morphotype. Persoonia 22:56-62. 